\title{
Change in Lifestyle pattern, behavior and Perception about Lockdown Amid COVID-19 Pandemic, India
}

\author{
Lockdown and its Effect, India \\ ${ }^{1}$ Kirti, ${ }^{2}$ Debarghya Mandal \\ ${ }^{1}$ Research Fellow, ${ }^{2}$ Data Analytics Consultant \\ ${ }^{1}$ Department of Biostatistics and Demography, \\ ${ }^{1}$ International Institute for Population Sciences, Mumbai, India \\ ${ }^{2}$ Sterlite, EdIndia, Mumbai, India
}

\begin{abstract}
Background: Since December 2019, the world has started to suffer an outbreak of Coronavirus disease (COVID19). Many people associated with community medicines affirmed that lockdown could help in abating the spread. However, this lockdown has changed our daily life habits. This proposed study will strive to observe this unprecedented changes. Aim: To assess the change in behavior or lifestyle among different segments and to understand different opinions and their association with social and psychological changes. Methodology: This study is based on a primary data that has been collected by circulating an online. Questions regarding the lifestyle pattern and behavioral change during the lockdown, perception and awareness about the pandemic has been asked. Tableau and MS-Excel has been used to analyze and present the results in an effective way. Results and Conclusions: Lockdown has its great effects on Internet Usage, workout routine, sleeping pattern, food intake and other lifestyle patterns. The level of awareness is different due to different exposures. Most people were supporting lockdown to stave off COVID-19 even at the cost of their daily bread. The social media can be seen as an integral part of this radical change. People are desperately striving to cope with the 'new normal'.
\end{abstract}

Index Terms: COVID-19; pandemic; lockdown; social distancing; lifestyle pattern; behavior; new normal; India

\section{INTRODUCTION}

The entire world is absolutely grappled with the pandemic of COVID-19 right now and an unprecedented bewilderment have engulfed our globe. The researchers have been trying to invent remedial measures; but all in vain. However, the virologists, the epidemiologists and the doctors associated with community medicines affirmed that lockdown could be one of the most effective tools to stave off the pandemic to some extent as, Social distancing can abate the infection undoubtedly. Since December 2019, the world has started to suffer an outbreak of Coronavirus disease (COVID-19). COVID-19 is an infectious disease caused by a newly discovered coronavirus (World Health Organization).

The disease spreads primarily through droplets of saliva or discharge from the nose when an infected person coughs or sneezes (World Health Organization). In order to prevent this inevitable disease from spreading the whole world's human population has been under a locked down. As a result, each and every individual in the world is facing some or the other problem as its consequences. The lockdown was activated on from the 24th of March 2020 and till date the whole country is in captivity literally. Needless to say that this lockdown has changed our habitual daily life radically and has crippled us socially, environmentally and psychologically. This proposed study will strive to observe this unprecedented changes with an eagle eye.

Some studies that has been published have reported the symptoms of the disease and its causes also the forecast of the spread of COVID-19 (Paital et. al., 2020, Roy Shovonlal, 2020, Zhang et. al., 2020). A paper which talks about the impact of lockdown, contact and non-contact transmissions on infection dynamics which is primarily based on the epidemiology of the disease and shows that the two different routes of transmission one is the traditional person-to-person contact route and the other one is formites-mediated and airborne route. The latter one is supposed to affect the spread if remain uncertain. Henceforth, premature withdrawal of lockdown is likely to promote a rapid and sharper increase in the number of new cases than the capacity of national; health-care services (Roy Shovonlal, 2020).

However, few studies have tried to ascertain the immediate impact of the lockdown amid COVID-19 pandemic on individual's mental health, lifestyle habits and their behavior. A study that has been done in Hubei Province of China among adults has shown that there are some positive impacts of the pandemic as the place has been under a locked down and people were paying more attention to their mental health, spending more time relaxing, resting and exercising (Zhang et. al., 2020). It has been observed that social lockdown has also drastic impacts on the environment especially on reduction of NO2 and $\mathrm{CO} 2$ emission. Therefore, the stringent social distancing via lockdown is highly important to control COVID-19 and also to contribute for self-regeneration of nature (Paital et. al., 2020). Sentiment analysis has shown that there is some negativity, fear, disgust, and sadness about the lockdown among the Indian population but the positive sentiments stood out (Barkur et. al., 2020). Only lockdown without mass testing is only postponing the surges, as transmission rate is getting reduced. If cases remained undetected then the transmission will resume (Chandrashekhar, 2020).

In Today's world where people likes to mingle with their family and friends, it becomes difficult for them being under a social lockdown and follow social distancing, where one cannot get out of the house until necessary. Present study majorly focusses on the awareness, perception about the COVID-19 outbreak, and to ascertain the immediate impact of the lockdown on change in individual's lifestyle habits and behavior during the lockdown amid COVID-19 pandemic in India. 


\section{DATA AND METHODS}

Data has been collected by circulating an online form through social media. People were asked to fill in the form by answering various questions regarding the lifestyle pattern and behavioral change during the ongoing lockdown, their perception and basic awareness about the COVID-19 pandemic etc. Anonymity of the responses has been maintained throughout the study. Respondents were asked about their age and profession for insightful information. Preliminary data points have been collected, asking if they are living with their family or not, if people are facing problem in getting the daily essentials, if people abide by the rules and regulations of lockdown enforced by the government and is able to cope with the prevailing situation in the country. Awareness about the situation, and few questions on how their lifestyle habits and pattern is being changing during the lockdown. There were in total eighteen questions in order to get the insights about the same among the population. Total of 530 sample points were collected from almost all the states of India between 14th of April till 24th of April. Almost all the regions have been covered, with most of the respondents from West Bengal and rest from other parts of the nation.

For analysis purpose various charts has been deployed to get the maximum and useful insights. Charts like bar graph, stacked bar graph, pie chart, donut chart has been used. Tableau and Microsoft Excel has been used to do the visualization and data cleaning respectively. Bar graph is a chart that uses bars and makes it easy to compare categories visually, where 100 percent stacked bar chart can be used to show the relative percentages of multiple data series in stacked manner. However, pie charts have been used to compare the contribution of each value to a total. Whereas, doughnut charts can be used when we want to show more than one dimension.

\section{RESULT AND DISCUSSION}

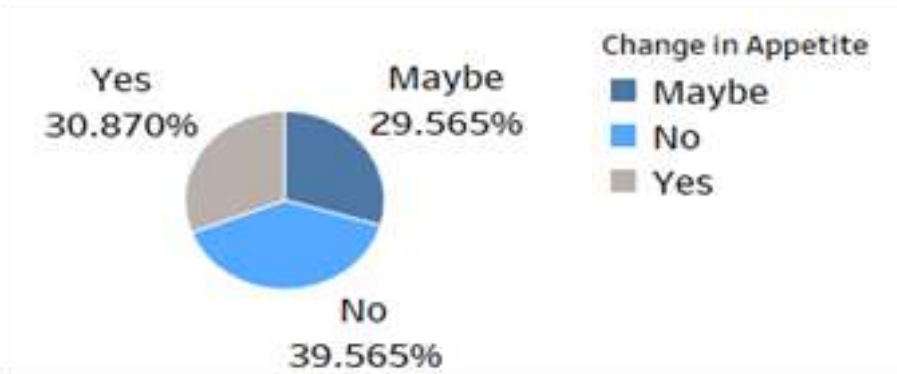

Figure 1.1: Overall Change in Appetite

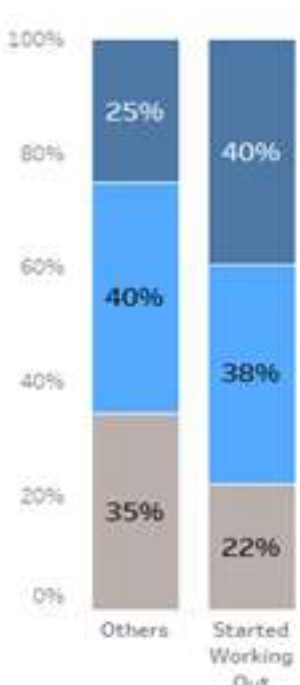

Out

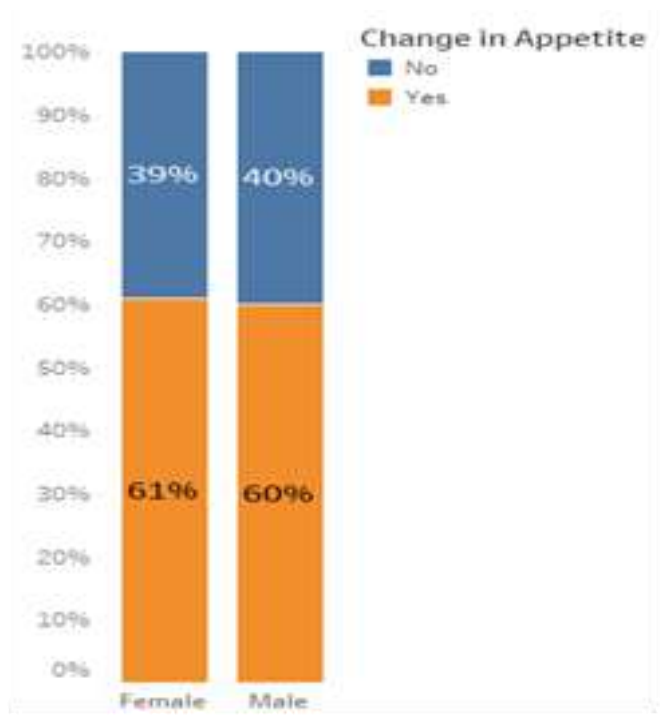

Figure 1.2: Change in Appetite by Gender

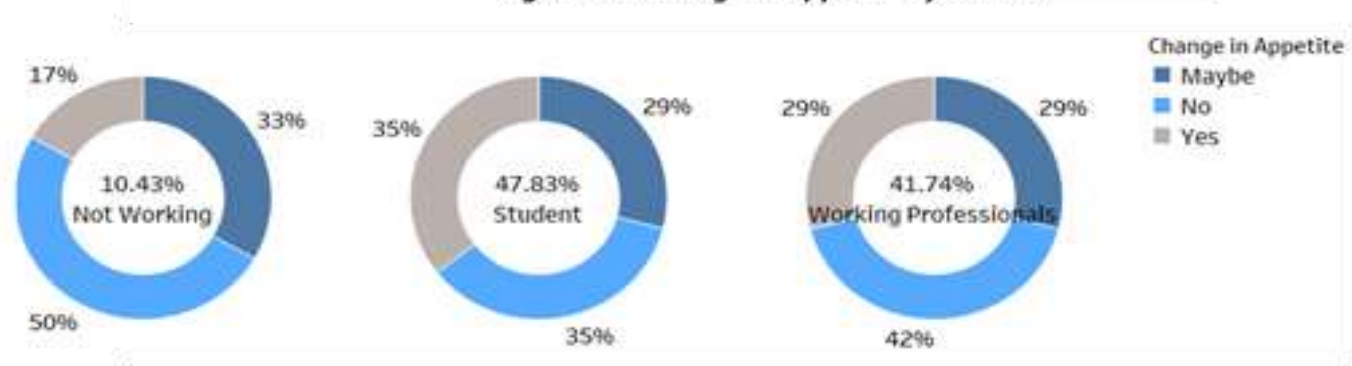

Figure 1.4: Change in Appetite by Profession

Figure 1.3: Change in Appetite Among those who has Started Working Out

From "Figure 1.1", approximately, 61 percent of the respondents have witnessed change in appetite during the lockdown. Also, those who has reported "May be" as their response were considered along with those who has reported "Yes" as they have experienced some amount of change in their appetite. "Figure 1.2" shows that pattern of change in Appetite is almost the same for both the gender. Most of the members be it male or female with approximately 60 percent each has experienced a change in Appetite. 
"Figure 1.3" depicts that among those who have started working out during the lockdown has approximately 62 percent of those who have experienced a change in appetite whereas among others there are approximately 60 percent people who have experienced a change in Appetite during the lockdown. From "Figure 1.4", we can see that the irrespective of the working status most of the respondents have witnessed a change in their appetite. However, Students and Working Professionals has shown a significantly greater proportion of people with change in appetite as compared to those who are Not Working.

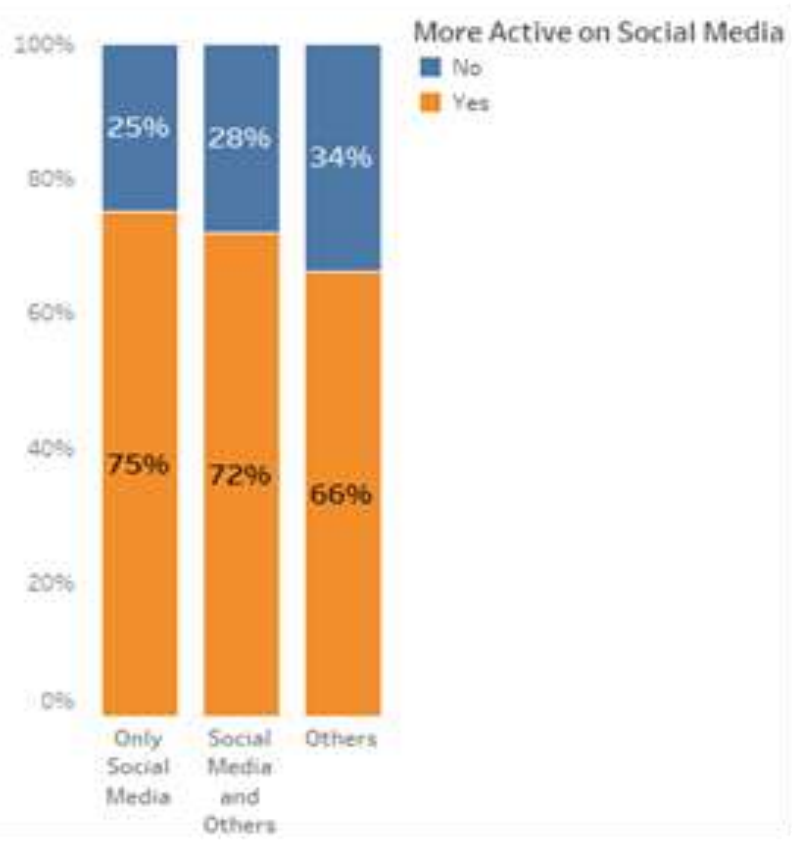

Figure 2: Social Media Use and if $\mathbb{R}$ has Increased

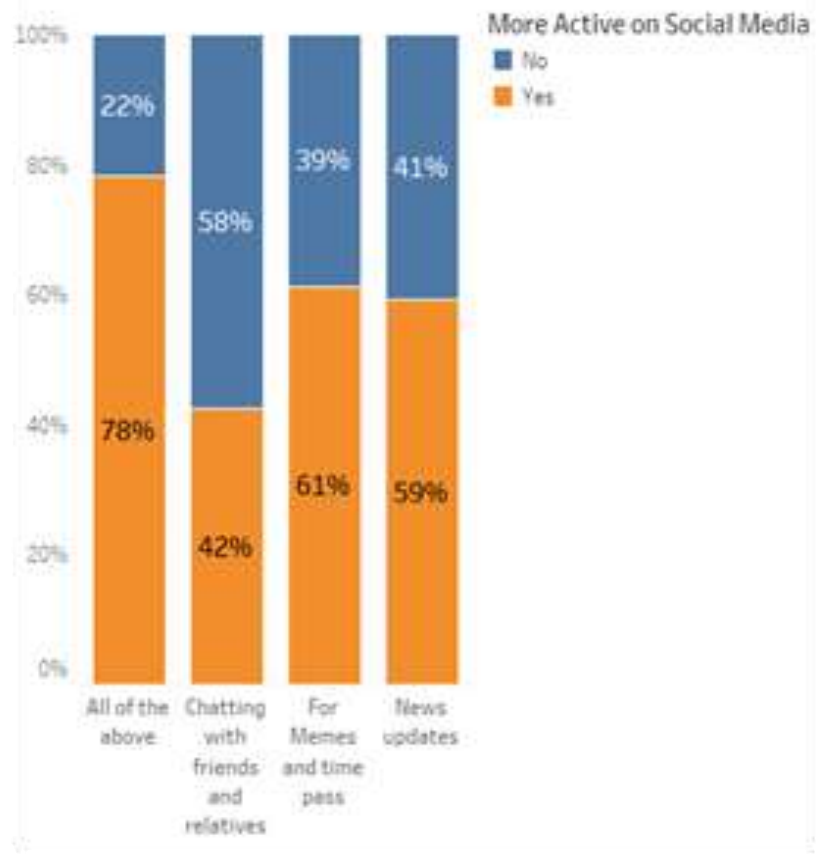

Figure 3: More active on Social Media and Purpose

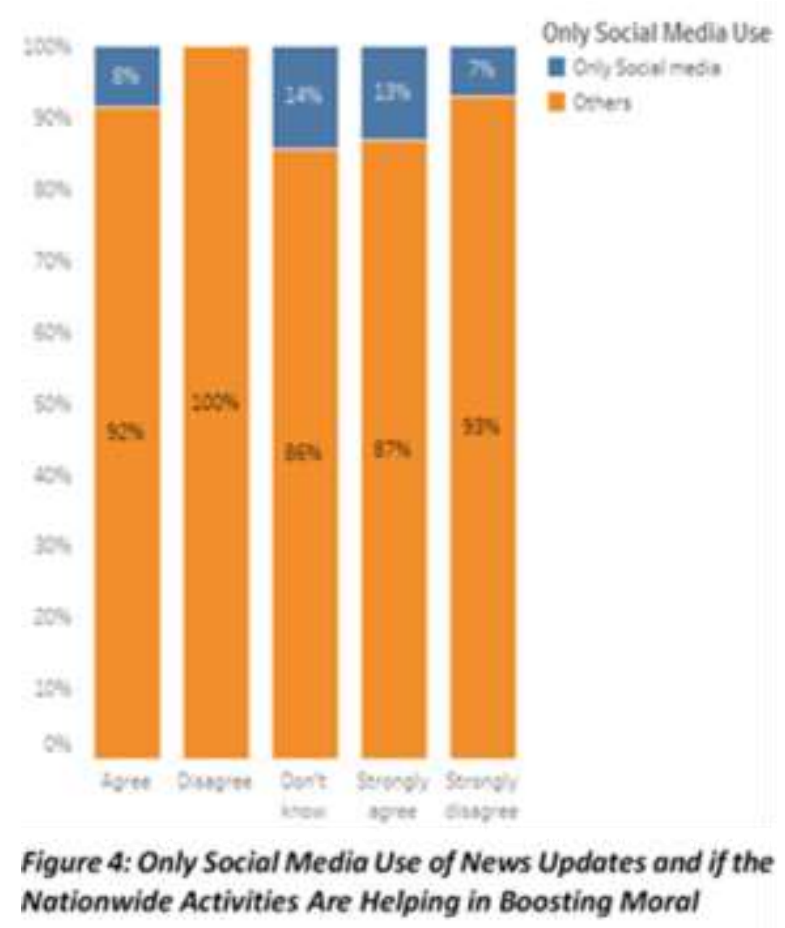

"Figure 2" depicts that those people who use Only Social media for COVID-19 statistics and news updates, a significant proportion of them are now more active on Social Media. Figures also shows that, most of the people are more active on Social Media during the Lockdown than before. From the "Figure 3", we can see that a big chunk of the sample i.e. 78 percent of the respondents are using Social Media for almost all the purposes like, chatting with Friends and Family Members, for Memes and Time pass and News Updates. Also, we can see that most of the people are who are now more active on Social Media use it only for the purpose of Memes and Time pass with 61 percent, which is even greater than those who are using it for News Updates.

From "Figure 4", we can see that, 100 percent people who use only Social Media for News updates has shown 'Disagreement' with the statement that 'the Nationwide activities such as clapping as expression of solidarity with those combating the COVID-19 
Pandemic, lighting diyas/candles/mobile torch etc. is actually helping in boosting moral'. Also, 93 percent of the respondents who only use Social Media for the News Updates 'Strongly Disagree' with the statement.

Which shows, that Social Media plays a vital role in molding or helping people in making their opinion about whatever is happening around them.

From "Figure 5.1", we can see that 64 percent of the respondents has reported that there are no positive cases nearby, 20 percent has responded "Yes" up till 24th of April i.e., the last survey date. Still there were 16 percent of the respondents who were unaware and has reported "Don't Know", rest with 84 percent of the respondents were aware of the situation in their locality.

"Figure 5.2" shows that, overall, most of the people agree or strongly agree with the statement that the Nationwide activities are helping in boosting moral with approximately 53 percent of the respondents and total of 31 percent are those who Disagree or Strongly Disagree with the statement. From "Figure 5.3", we can see that irrespective of the State a significant proportion of people have noticed a positive environmental change with approximately 68 percent in West Bengal and 71 percent in Others states. Whereas, 18 percent of the respondents have not witnessed any change in environment in West Bengal and only 10 percent in Other States. "Figure 5.4" depicts that approximately, 70 percent of the respondents has experienced a positive environmental change in their respective areas. From "Figure 5.5" we can see that, 50 percent of the respondents are not facing any problem in accessing grocery items, 19 percent people are saying that they are facing problem in getting the grocery items. Whereas, 31 percent are saying that in near future if the satiation does not get settled then there might be a problem in accessing grocery items.

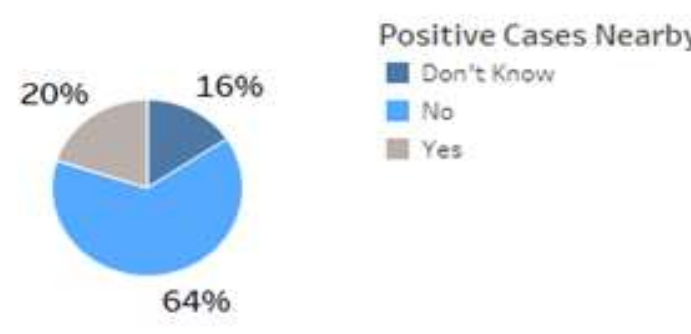

Figure 5.1: People Having Positive Cases Nearby

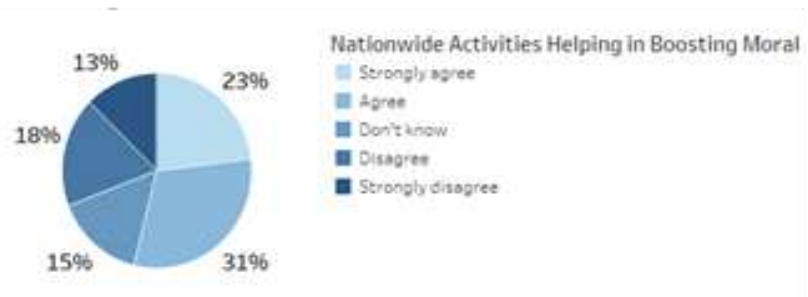

Figure 5.2: Opinion if Nationwide Activities Helping in Boosting Moral

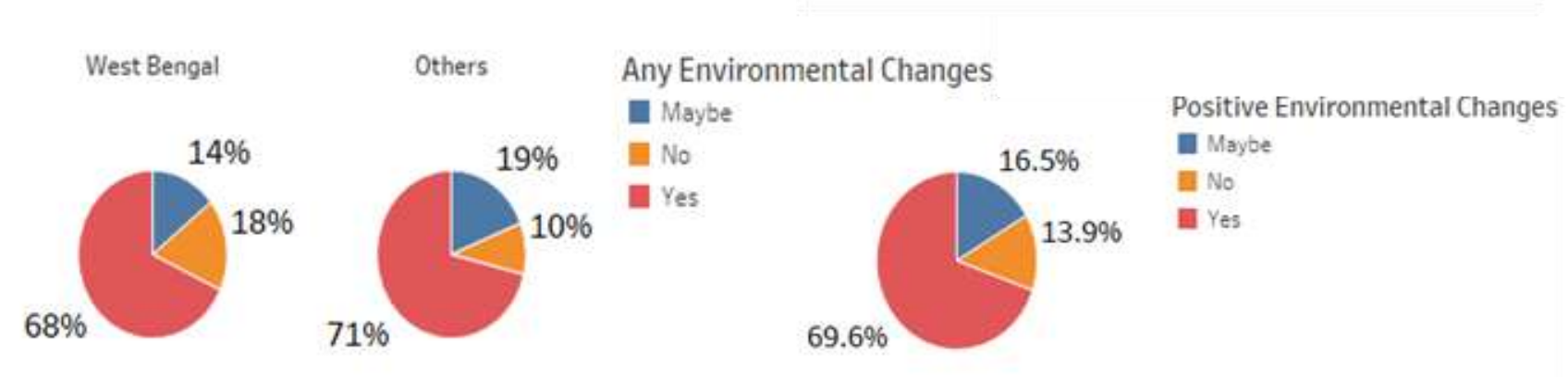

Figure 5.3: Environmental Change by West Bengal and Others

Figure 5.4: If there is any Positive environment change

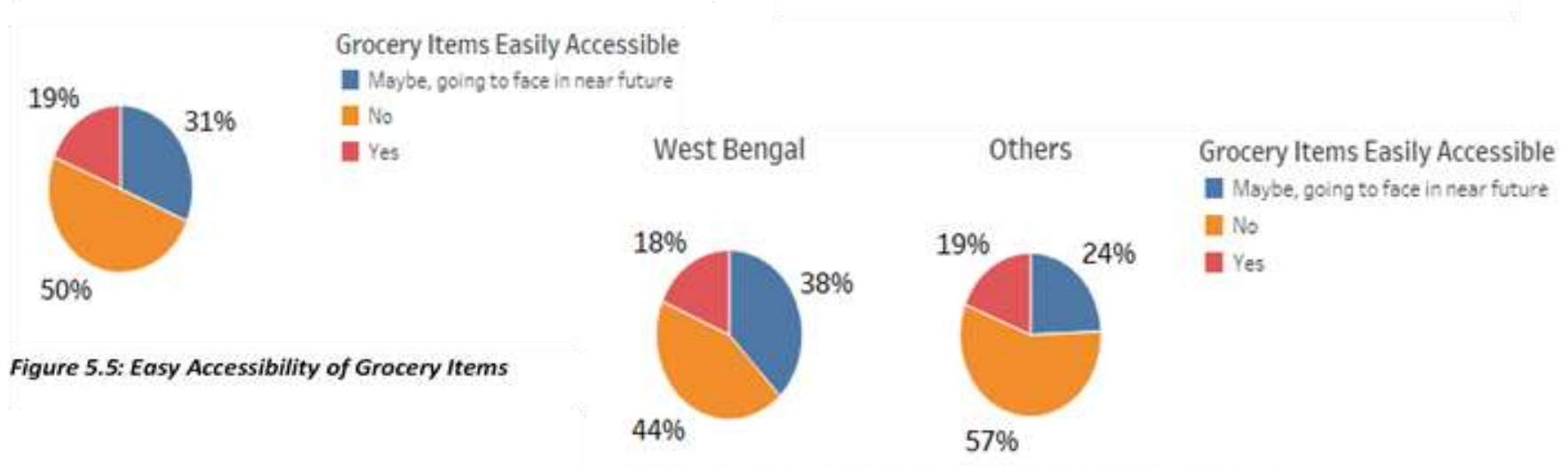

Figure 5.6: Grocery Item Accessibility across West Bengal and Others 


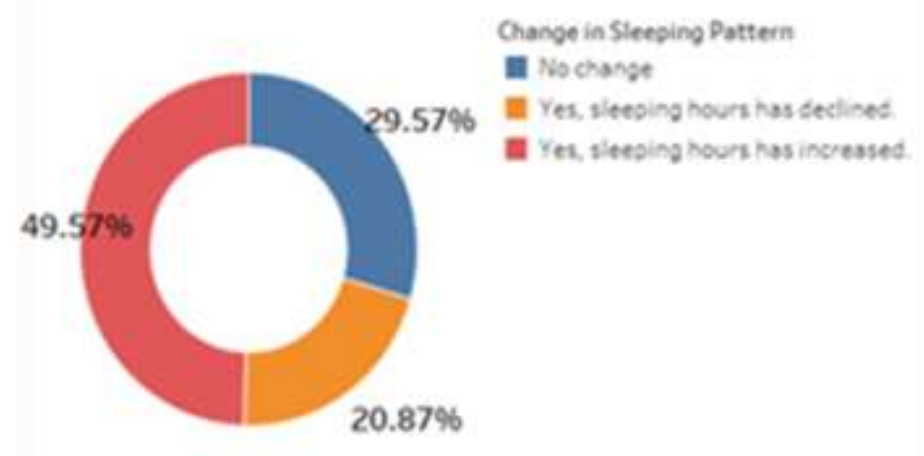

Figure 6: Change in Sleeping Pattern During the Lockdown

From "Figure 6" we can see that, almost half of the respondents have experienced an increase in sleeping hours during the lockdown as compared to pre lockdown sleeping hours. From the above "Figure 7", we can see that most of the respondents have experienced an increase in the sleeping hours irrespective of the profession during the lockdown as compared to the sleeping hours pre lockdown. Among students an increased sleeping hours can be seen which is the highest amongst all with 56 percent. Those who are working professionals, most of them have experienced an increase in the sleeping hours. Whereas, for those who are not working 50 percent of them is having the same sleeping pattern as pre lockdown hence, there is no change in sleeping pattern. 


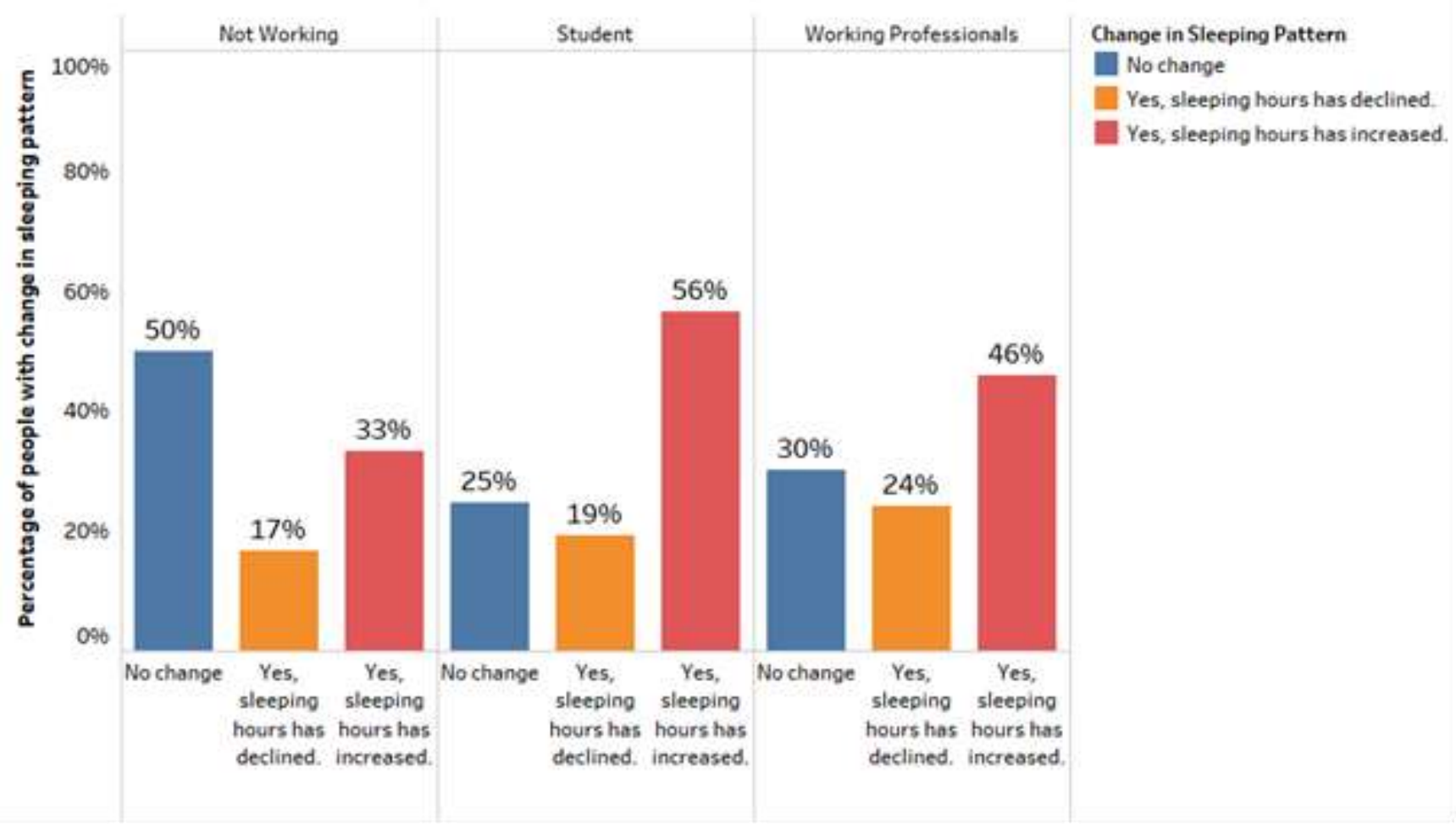

Figure 7: Sleeping Pattern by Profession

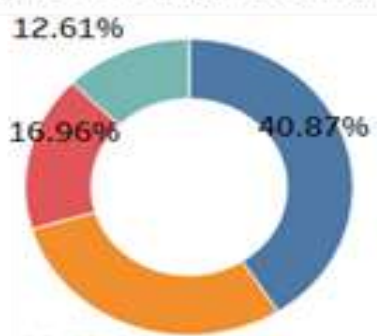

$29.57 \%$

Figure 8.1: Working Out During the Lockdown

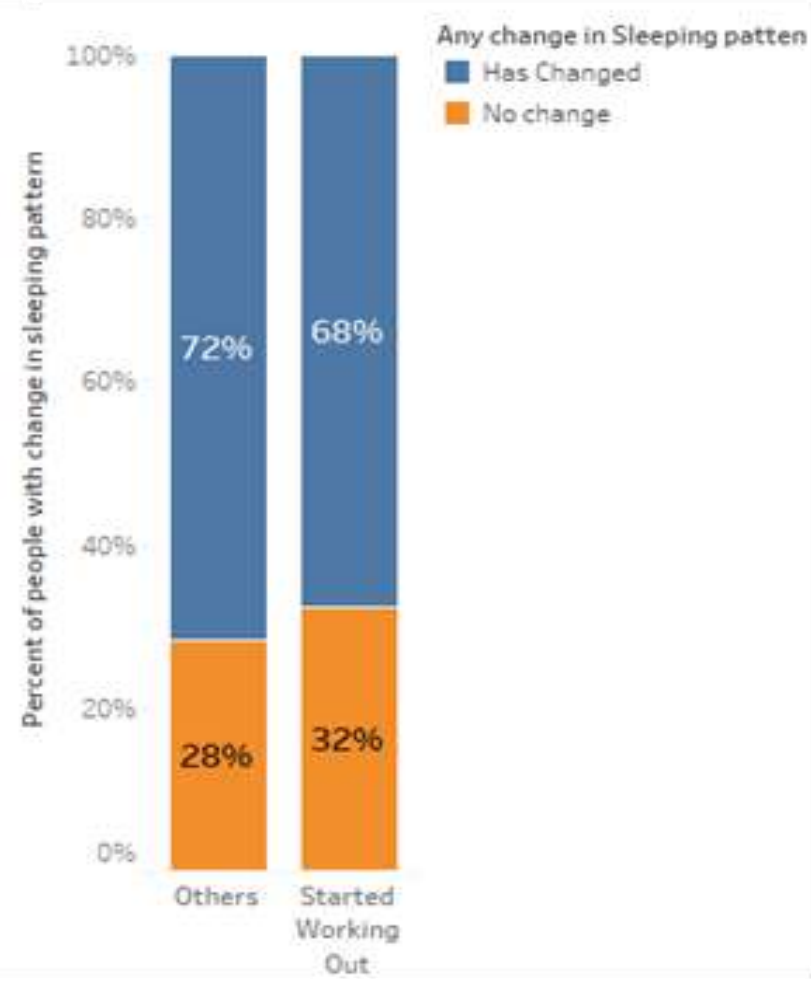

Figure 8.3: Started Working Out and Change in Sleep Pattern

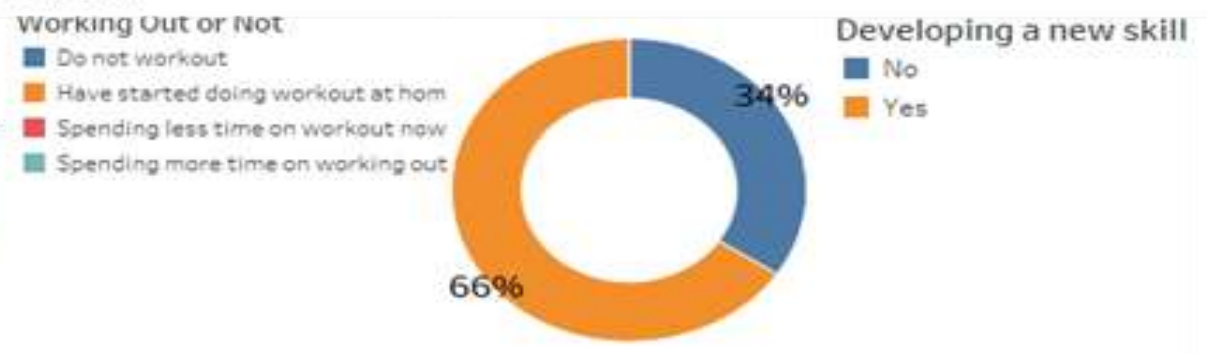

Figure 8.2: People Who Have Started Developing a New Skill/s

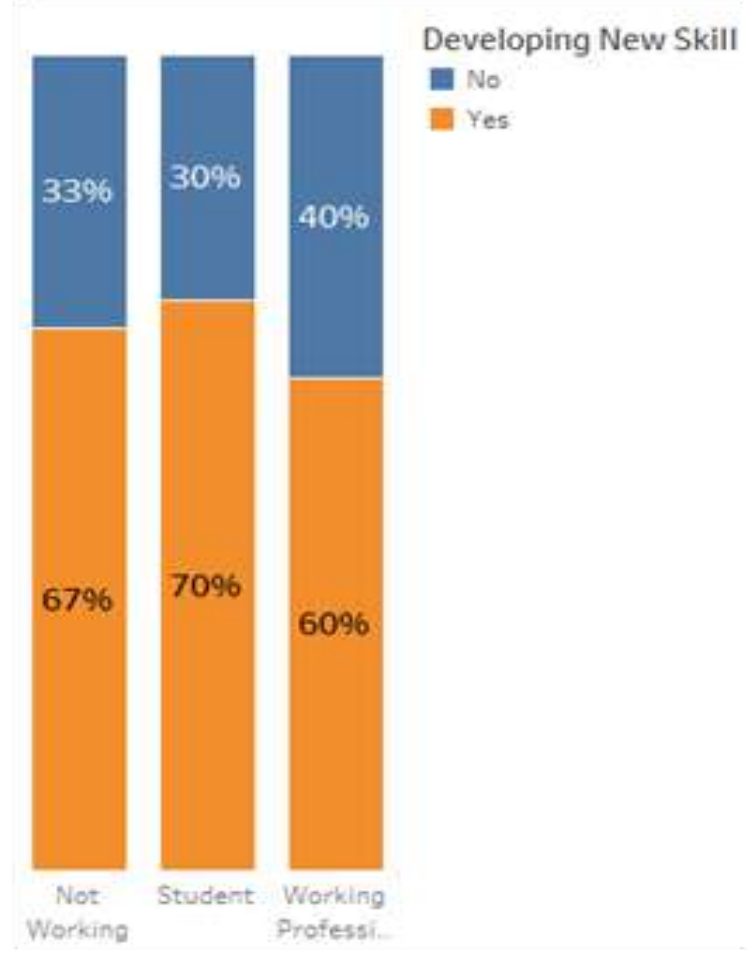

Figure 8.4: People Who Have Started Developing a New Skill/s by Profession 
"Figure 8.1" depicts that approximately 30 percent people have started working out during the lockdown. Also, during the lockdown the number of those who do not workout has dropped down by 30 percent, henceforth, the number of those who work out has doubled during the lockdown. From "Figure 8.2" we can see that 66 percent of the respondents have started to work on developing a new skill/s during the lockdown and utilizing the period for self-development. From "Figure 8.3" we can see that those who have started working out during the lockdown the change in sleeping pattern experienced is comparatively less number than others though, among both the categories most of the people has experienced a change in their sleeping pattern during the lockdown. "Figure 8.4" shows that, 70 percent of the students have started working on developing new skill/s which is the highest followed by 67 percent of those who are not working have started to working on developing a new skill/s. However, those who are working professionals have comparatively less people who are working in the direction of developing a new skill with 60 percent.

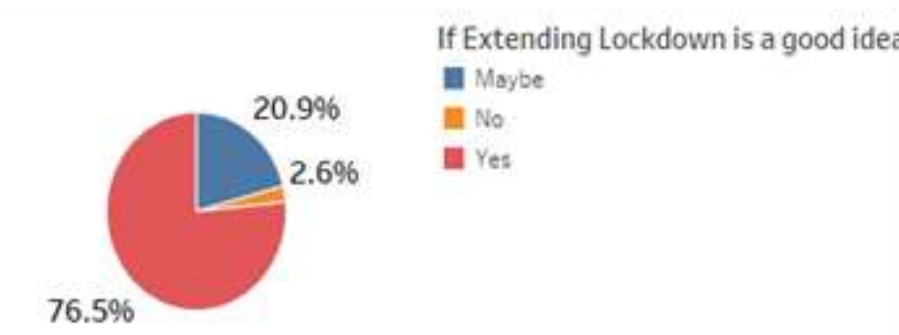

Figure 9: If extending Lockdown is a Good Idea
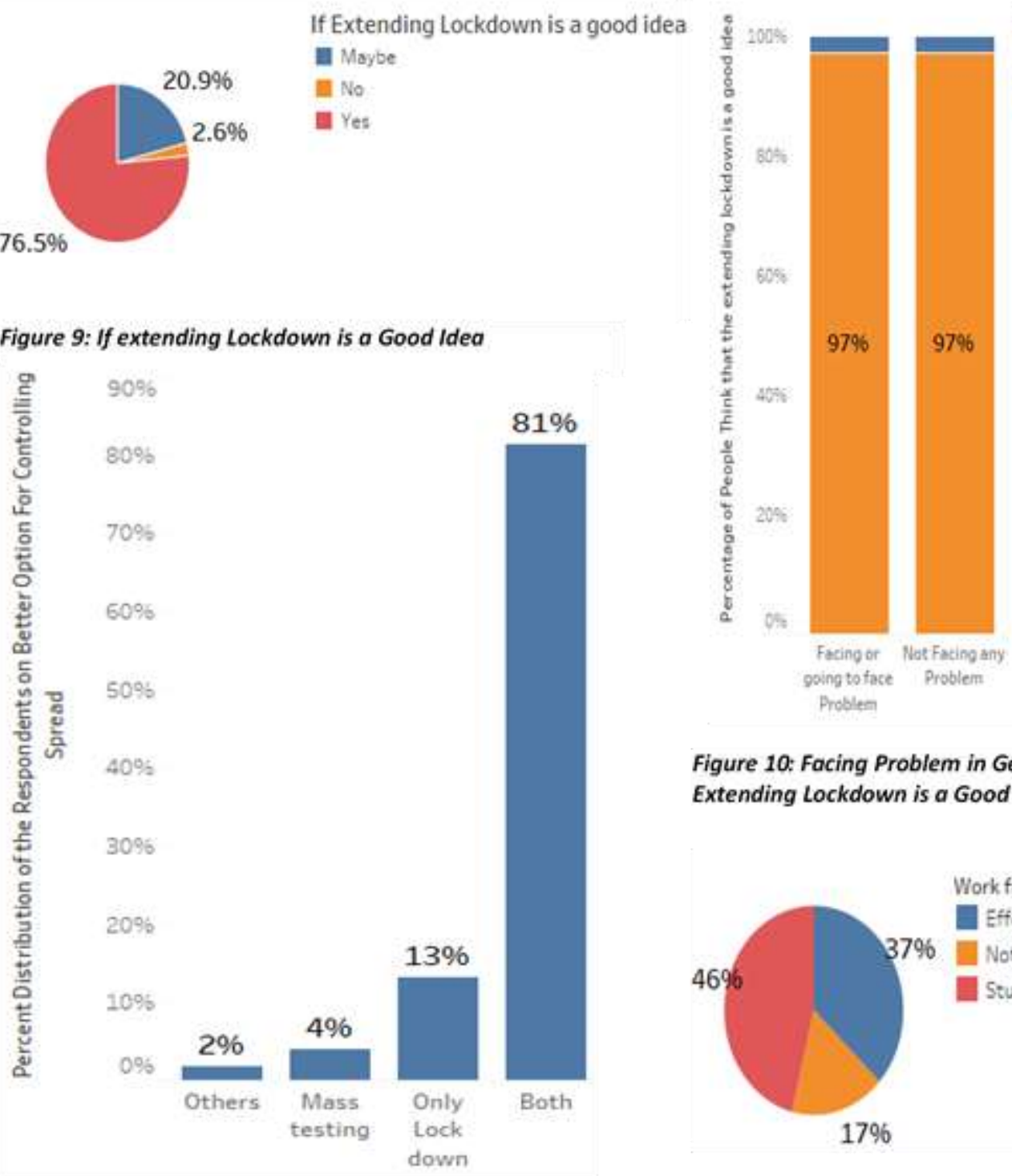

Extending lock down is a goodidea

No

Iyes

Figure 10: Facing Problem in Getting Grocery Item and If Extending Lockdown is a Good Idea

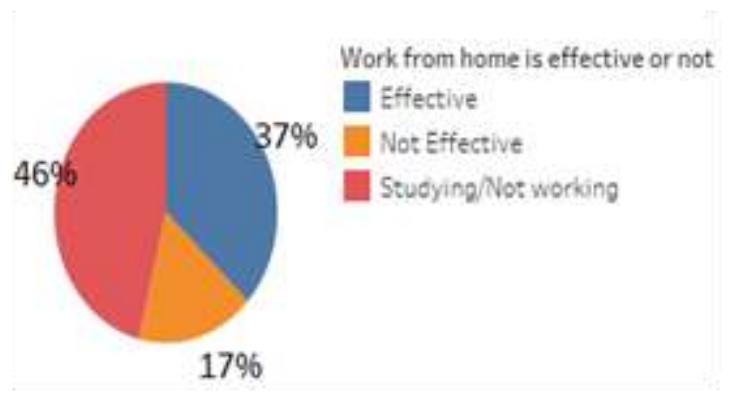

Figure 11: Respondents Opinion on Better Option for Controlling Spread

Figure 12: Work from home is effective or not

"Figure 9" show that, approximately 77 percent of the respondents are pro Lockdown whereas 21 percent are unsure about the decision. Only 2 percent respondents are against the decision. "Figure 10" shows that irrespective of whether a person is facing any problem in getting the grocery item or not most of them are in the favor of lockdown with 97 percent of the respondents.

From "Figure 11" we can see that 81 percent of the respondents suggest that both, lockdown as well as Mass testing are required if one wants to control the spread of COVID-19 disease followed by 13 percent people who think that only lockdown would help enough in controlling the disease spread. However, only 4 percent people think that only mass testing would be enough. From "Figure 12", it can be seen that more than half of those who are working says that work from home is effective with approximately 37 percent among all the respondents. 


\section{CONCLUSION}

Most people were supporting lockdown to stave off COVID-19 even at the cost of their daily bread and lifestyle. In fact, the lockdown has drastically changed the pattern of lifestyle and eventually our attitude towards life. It has changed people's food habit, pattern of workout, pattern of unwinding, pattern of social media usage, pattern of studying and working; needless to say all these things are correlated. Same results can be seen in many studies (Zhang and Zheng, 2020). The social media is an integral part this radical change. It should be kept in mind that social media can mold, modify one's idea and concept. It can shift one's point of view, can inspire to take decisions. This study shows the differences of thinking or opinions among different groups. Users identity social status, volume of usage of internet, specific interaction and people involved in the interactions, duration of usage contributed a lot in decision making. Anyway, people are desperately striving to cope with the 'new normal' and people will continue to do so even in post lockdown period.

\section{REFERENCES}

[1] Barkur, G., \& Vibha, G. B. K. (2020). Sentiment analysis of nationwide lockdown due to COVID 19 outbreak: Evidence from India. Asian journal of psychiatry.

[2] Chandrashekhar, V. (2020). 1.3 billion people. A 21-day lockdown. Can India curb the coronavirus. Science.

[3] Paital, B., Das, K., \& Parida, S. K. (2020). Inter nation social lockdown versus medical care against COVID-19, a mild environmental insight with special reference to India. Science of the Total Environment, 138914.

[4] Petropoulos, F., \& Makridakis, S. (2020). Forecasting the novel coronavirus COVID-19. PloS one, 15(3), e0231236.

[5] Roy, S. (2020). COVID-19 pandemic: Impact of lockdown, contact and non-contact transmissions on infection dynamics. medRxiv.

[6] World Health Organization. (2020). Coronavirus disease 2019 ( COVID-19): situation report, 85.

[7] Zhang, Y., \& Ma, Z. F. (2020). Impact of the COVID-19 pandemic on mental health and quality of life among local residents in Liaoning Province, China: A cross-sectional study. International journal of environmental research and public health, 17(7), 238 